\title{
Frecuencia de alteraciones de la huella plantar en escolares de una comunidad mexicana
}

\section{Frequency of footprints alterations in school children from a Mexican community}

\author{
Aco-Luna JA,* Rodríguez-Jiménez F, ${ }^{\ddagger}$ Guzmán-Coli MG,* \\ Enríquez-Guerra MA, ${ }^{\S}$ Chavarría-Bernardino IG*
}

Benemérita Universidad Autónoma de Puebla, Puebla, México.

RESUMEN. Introducción: Las alteraciones de la huella plantar en el niño es causa de preocupación en los padres de familia. Objetivo: Determinar la frecuencia de alteraciones de la huella plantar en escolares. Determinar si existe relación entre el sobrepeso y la obesidad con la presencia de alteraciones de la huella plantar. Material y métodos: Estudio observacional, transversal y prospectivo. Se evaluaron 959 escolares de seis a 13 años de edad. Se registró peso, talla, índice de masa corporal para la edad. La huella plantar se catalogó en pie plano y pie cavo utilizando el índice del arco. Para el análisis y comparación estadística se utilizó el programa SPSS versión 24 con las pruebas $\chi^{2}$ y ANOVA. Resultados: Se revisaron 530 niños (55.3\%) y 429 niñas (44.7\%). La media de edad fue de 8.97 años. Se observaron 182 niños (19\%) con alteración de la huella plantar, $42.3 \%$ con pie plano y $57.7 \%$ con pie cavo. Ninguno mostró sintomatología del pie. Ciento treinta y un niños tenían sobrepeso y 52 obesidad, sin influir en la presencia de alguna alteración de la huella plantar $(p=0.20)$. La relación de pie plano fue mayor en los hombres (2.5:1) y de pie cavo fue mayor en las mujeres (1.3:1). Conclusiones: En nuestro grupo de estudio se encontró mayor prevalencia del pie cavo en comparación con el pie plano. El peso corporal no influyó en las alteraciones de la huella plantar.

Palabras clave: Huella plantar, plano, cavo, sobrepeso, diagnóstico.

\section{Nivel de evidencia: IV}

* Alumno.

₹ Alumno. Academia de Musculoesquelético.

$\S$ Profesor.

Facultad de Medicina. Benemérita Universidad Autónoma de Puebla, Puebla, México.

Dirección para correspondencia:

Jesús Alberto Aco-Luna

Benemérita Universidad Autónoma de Puebla, Facultad de Medicina, Departamento de Genética 13 Sur 2702, Col. Volcanes, 72000, Puebla, Puebla, México.

Fax: +52 222 2295500, ext. 5013, Contacto: +52 2221053391

E-mail: jealaclu@gmail.com

Este artículo puede ser consultado en versión completa en: www.medigraphic.com/actaortopedica
ABSTRACT. Introduction: Alterations in the plantar footprint in the child are a cause for concern in parents. Objective: Determine the frequency of plantar footprint alterations in school children. Determine if there is a relationship between overweight and obesity with the presence of alterations of the plantar footprint. Material and methods: Observational, transversal and prospective study. 959 schoolchildren aged six to 13 were evaluated. Weight, size, body mass index for age were recorded. The plantar footprint was cataloged on flat foot and cavus foot using the arch index. For statistical analysis and comparison, the SPSS version 24 program was used with the $\chi^{2}$ and ANOVA tests. Results: 530 children (55.3\%) and 429 girls (44.7\%). The median age was 8.97 years. 182 children were found (19\%) with alteration of the plantar footprint, $42.3 \%$ with flat foot and $57.7 \%$ with cavus foot. None of them had foot symptoms. 131 children were overweight and 52 obese, without influencing the presence of any alteration of the plantar footprint $(\mathrm{p}=0.20)$. The flat-foot ratio was higher in men (2.5:1) and standing cavus major in women (1.3:1). Conclusions: In our study group we find a higher prevalence of the cavus foot compared to the flat foot. Body weight did not influence plantar footprint alterations.

Keywords: Plantar footprint, flat, cavus, overweight, diagnosis.

\section{Introducción}

El pie es la base de la sustentación del aparato locomotor. Se divide para su estudio en: bóveda plantar, talón y antepié. ${ }^{1}$ Las deformidades del pie pueden comprometer el eje transversal, vertical o longitudinal. ${ }^{2,3}$ El desarrollo del arco longitudinal medial del pie ocurre durante varios años con un gran espectro de variaciones normales. ${ }^{4}$

El pie plano se define como la deformación en la cual el arco plantar interno ha disminuido en su altura o ha desaparecido, generando un aumento de la huella plantar como resultado de alteraciones en la elasticidad ligamentaria, lo 


\begin{tabular}{|c|c|c|c|c|}
\hline \multirow[b]{3}{*}{ Edad } & \multicolumn{4}{|c|}{$\begin{array}{l}\text { Tabla 1: Población estudiada. } \\
\text { Edad y presencia de deformidad del pie. }\end{array}$} \\
\hline & \multicolumn{3}{|c|}{ Tipo de pie } & \multirow[b]{2}{*}{$\begin{array}{c}\text { Total } \\
(\mathrm{p}=0.016)\end{array}$} \\
\hline & $\begin{array}{c}\text { Pie } \\
\text { normal }\end{array}$ & $\begin{array}{l}\text { Pie } \\
\text { plano }\end{array}$ & $\begin{array}{l}\text { Pie } \\
\text { cavo }\end{array}$ & \\
\hline 6 & 118 & 8 & 9 & 135 \\
\hline 7 & 116 & 8 & 7 & 131 \\
\hline 8 & 114 & 17 & 17 & 148 \\
\hline 9 & 124 & 11 & 24 & 159 \\
\hline 10 & 113 & 14 & 26 & 153 \\
\hline 11 & 61 & 3 & 11 & 75 \\
\hline 12 & 123 & 14 & 10 & 147 \\
\hline 13 & 8 & 2 & 1 & 11 \\
\hline Total & 777 & 77 & 105 & 959 \\
\hline
\end{tabular}

que condiciona desequilibrio muscular del pie. ${ }^{2,5}$ Se atribuye a la hiperlaxitud ligamentaria y al sobrepeso. La obesidad ha sido considerada un factor de riesgo de presentar pie plano. ${ }^{6,78}$ Se divide en pie plano flexible, flexible con talón de Aquiles corto y rígido. ${ }^{9}$

La prevalencia de pie plano (flexible) en niñas/niños de dos a seis años varía de 21 a $57 \%$ y disminuye de 13.4 a $27.6 \%$ en la edad escolar y persiste en $3 \%$ en la edad adulta. ${ }^{2,10}$

El pie cavo se caracteriza por aumento de altura del arco longitudinal medio (arco alto) asociado a menudo a varo del retropié. Puede clasificarse como fisiológico o neuromuscular. ${ }^{11,12}$ La edad de presentación se encuentra entre los ocho y 12 años. ${ }^{2}$

$\mathrm{Al}$ momento existen pocos artículos que investiguen la presencia de pie plano y pie cavo simultáneamente, por lo que es conveniente su búsqueda en nuestra población. ${ }^{5,6,7,8,13}$ Nuestro objetivo es conocer la frecuencia de alteraciones de la huella plantar en una población mexicana específica; evaluar en este grupo si existe mayor frecuencia de alguna alteración del pie ante sobrepeso u obesidad y mencionar si existe disminución de la presencia de alteración de la huella plantar ante el aumento de la edad del educando.

\section{Material y métodos}

El presente estudio es de tipo observacional y prospectivo. Se evaluaron 980 niños de dos escuelas de nivel primara en la comunidad de Cacalotepec, Puebla, México, catalogada como de alta marginación. ${ }^{14}$ Se solicitó autorización a las instituciones educativas y a su vez a los padres de familia, se mencionó la técnica de este estudio y los procesos a realizar. La población estudiada no fue sometida a ninguna prueba experimental. Se evaluaron las variables sexo, edad, peso, talla, índice de masa corporal, alteración de la huella plantar.

Para el peso se realizó la medición a través de una báscula con resultado en kilogramos, la talla fue expresada en metros con el uso de un estadímetro. Una vez obtenidos los datos se evaluó el índice de masa corporal con la fórmula de la Organización Mundial de la Salud acorde a la tabla de IMC para la edad. ${ }^{15}$ Una vez obtenidos los percentiles fueron clasificados en cuatro grupos, (bajo peso, peso normal, sobrepeso y obesidad).

Para la obtención de la huella plantar se utilizó un pedígrafo convencional sobre el cual los niños colocaban un pie en bipedestación. Una vez obtenidas las impresiones fueron clasificadas utilizando el índice de arco (IA) (calculado como la relación entre el área del tercio medio de la huella y toda el área de la huella) catalogando en pie cavo (IA < $21 \%$ ), pie normal (IA $21-26 \%$ ) y pie plano (IA $>26 \%$ ). ${ }^{16}$

Los criterios de eliminación del estudio fueron: edad menor de seis años y mayor de 13 años; presentar dolor o alguna lesión visible en rodilla o piernas al momento del estudio; no acudir durante los días del estudio. Se excluyeron 21 niños y un total de 959 niños $(n=959)$ fueron evaluados y se obtuvieron las variables a estudiar. Todas las variables fueron analizadas con el programa SPSS 24.0 y se utilizó la prueba $\chi^{2}$ para la correlación de las variables.

Una vez terminada la búsqueda y la clasificación, se creó un formato con las recomendaciones acerca del calzado indicando que no existen zapatos correctivos, sólo para su protección. ${ }^{17}$ Además, se hizo mención de que la valoración era requerida sólo si el niño refería dolor al realizar la pisada en los casos de pie cavo.

\section{Resultados}

Se evaluaron 530 niños (55.3\%) y 429 niñas (44.7\%), con una edad mínima de seis años y máxima de 13 años, media de $8.97 \pm 2.0$ (Tabla 1).

Se catalogaron los niños en bajo peso (percentil menor de cinco) 110 niños (11.5\%), peso normal (percentil mayor de cinco y menor de 85) 666 niños (69.5\%), sobrepeso (percentil 85 menor de 95) 131 niños (13.7\%) y obesidad (percentil igual o mayor de 95) 52 niños (5.4\%).

Ciento ochenta y dos niños $(\mathrm{n}=182) 19 \%$ mostraron alteraciones de la huella plantar, 77 (42.3\%) con pie plano y 105 con pie cavo (57.7\%), ( $<0.05)$. Ante la presencia de pie plano se encontraron 55 niños y 22 niñas; y escolares con pie cavo fueron 45 niños y 60 niñas ( $<<0.05$ ).

\begin{tabular}{|c|c|c|c|c|c|}
\hline \multirow[b]{2}{*}{ Tipo de pie } & \multicolumn{4}{|c|}{ IMC para la edad } & \multirow[b]{2}{*}{ Total } \\
\hline & $\begin{array}{l}\text { Bajo } \\
\text { peso }\end{array}$ & $\begin{array}{l}\text { Peso } \\
\text { normal }\end{array}$ & Sobrepeso & Obesidad & \\
\hline Pie plano & 6 & 50 & 12 & 9 & 77 \\
\hline Pie cavo & 19 & 79 & 6 & 1 & 105 \\
\hline Pie normal & 85 & 537 & 113 & 42 & 777 \\
\hline Total & 110 & 666 & 131 & 52 & 959 \\
\hline \multicolumn{5}{|c|}{$\chi^{2}=20.812 \mathrm{Gl}=6, \mathrm{p}=0.002$} & \\
\hline
\end{tabular}


En relación con la presencia de alteración de la huella plantar, en comparación con el estado nutricional del educando, en nuestra población se observó que el estado nutricio no influye en la presencia de alteraciones de la huella plantar, ya que se detectaron más casos de deformidades en niños con IMC normal (mayor de cinco y menor de 85 ) que en sobrepeso u obesidad $(\mathrm{p}=0.002)($ Tabla 2$)$.

\section{Discusión}

Se encontró que 19\% de los niños tienen una deformidad de la huella plantar. En la población estudiada hay mayor frecuencia de pie cavo (57.7\%) en comparación con la presencia de pie plano (42.3\%).

La presencia de alteración de la huella plantar fue mayor en niños (10.4\%) que en niñas (8.6\%). En relación al estado nutricional, en nuestro grupo de estudio el IMC mayor que el percentil 85 catalogado como sobrepeso no tuvo influencia en el desarrollo de una alteración de la huella plantar $\left(\chi^{2}\right.$ $=2.31, \mathrm{p}=0.16)$, del mismo modo, el percentil mayor de 95 equivalente a obesidad $\left(\chi^{2}=0.02, \mathrm{p}=0.87\right)$.

Se observó que la presencia de pie plano fue de $8 \%$ en comparación con Pfeiffer M y colaboradores, ${ }^{10}$ quienes evaluaron 835 niños de un grupo de edad de tres a seis años con una prevalencia de $44 \%$. La presencia de pie plano fue de $52 \%$ en niños y de $36 \%$ en niñas. Y con una reducción significativa con la edad aproximadamente de $36.8 \%$ por año. Nuestro estudio reveló que la edad con prevalencia más alta de pie plano fue a los ocho años con $22.1 \%$ y con una disminución gradual por año de $14.3 \%$.

González de Aledo LA y colegas ${ }^{18}$ en un estudio con 948 niños de cuatro a 13 años de edad reportaron 155 niños (16.3\%) con pie cavo. En este estudio se observó 10.9\% (105) con pie cavo.

Espinoza-Navarro O y su equipo ${ }^{19}$ realizaron la valoración de la huella plantar a 420 niños de seis a 12 años de edad y encontraron 171 escolares con deformidades de la huella plantar, $28 \%$ con pie plano y $13 \%$ con pie cavo, lo que dista de los resultados expuestos en esta revisión, puesto que se detectó mayor tendencia de pie cavo que de pie plano.

En población mexicana, Saldívar-Cerón HI y colaboradores $^{7}$ evaluaron en Tamaulipas, México 1,128 estudiantes en edad escolar de nueve a 11 años de edad y observaron $12.1 \%$ de su población con pie plano, comparada con nuestra población fue menor, afectó a 8\% de los escolares evaluados.

\section{Conclusión}

En nuestra población se encontró mayor prevalencia de pie cavo que de pie plano. El sobrepeso y la obesidad no influyeron en la presencia de alguna alteración de la huella plantar.

\section{Agradecimientos}

Se agradece la participación de Rosa María Aldana Armas, Elías Castro Jiménez y Yesenia Largo González por su colaboración en la realización del presente estudio.

Consideraciones éticas: Este protocolo se ajusta a los lineamientos de la Ley General de Salud de México promulgada en 1986 y al código de Helsinki de 1975 y modificado en 1989, respecto a la confidencialidad de los participantes en el estudio. El estudio que nos ocupa es de tipo observacional y prospectivo, por lo que los sujetos estudiados no fueron sometidos a ninguna prueba experimental.

Bibliografía

1. Voegeli AV. Anatomía funcional y biomecánica del tobillo y el pie. Rev Esp Reumatol. 2003; 30(9): 469-77.

2. Moya H. Malformaciones congénitas del pie y pie plano. Rev Chil Pediatr. 2000; 71(3): 243-5.

3. Muñoz J. Deformidades del pie. An Pediatr Contin. 2005; 4(4): 251-8.

4. Carr JB, Yang S, Lather LA. Pediatric pes planus: a state-of-the-art review. Pediatrics. 2016;137(3) 1-10

5. Aldaco-García VD, et al. Guía de Práctica Clínica Abordaje diagnóstico del Pie Plano en Niñas/Niños y las/los Adolescentes en el primer nivel de atención. 2015. CENETEC. Instituto Mexicano del Seguro Social. 1-39.

6. Sadeghi-Demneh E, Azadinia F, Jafarian F, Shamsi F, Melvin JM, Jafarpishe M2, Rezaeian Z. Flatfoot and obesity in school-age children: a cross-sectional study. Clin Obes. 2016; 6(1): 42-50.

7. Saldívar-Cerón HI, Garmendia-Ramírez A, Rocha-Acevedo MA, Pérez-Rodrígueza P. Obesidad infantil: factor de riesgo para desarrollar pie plano. Bol Med Hosp Infant Mex. 2015; 72(1): 55-60.

8. Jankowicz-Szymanska A, Mikolajczyk E. Effect of excessive body weight on foot arch changes in preschoolers. J Am Podiatr Med Assoc. 2015; 105(4): 313-9.

9. Zafiropoulos G, Prasad KS, Kouboura T, Danis G. Flat foot and femoral anteversion in children. A prospective study. Foot. 2009; 19: 50-4.

10. Pfeiffer M, Kotz R, Ledl T, Hauser G, Sluga M. Prevalence of flat foot in preeschool-aged children. Pediatrics. 2006; 118(2): 634-9.

11. Walter KD. Capítulo 201 Pie. En: Nelson, Marcdante KJ, et al. Pediatría esencial. Elsevier Mosby, 2012. pp. 279-82.

12. Larrosa-Padró M, Mas-Moliné S. Alteraciones de la bóveda plantar. Rev Esp Reumatol. 2003; 30(9): 489-98.

13. Banwell HA, Paris ME, Mackintosh S, Williams CM. Paediatric flexible flat foot: how are we measuring it and are we getting it right? A systematic review. J Foot Ankle Res. 2018; 11: 21.

14. CONAPO. Índice de marginación por localidad 2010. Disponible en: http://www.microrregiones.gob.mx/catloc/contenido. aspx?refnac $=211190032$

15. De Onis M, Onyango AW, Borghi E, Siyam A, Nishida C, Siekmann J. Development of a WHO growth reference for school-aged children and adolescents. Bull World Health Organ. 2007; 85(9): 660-7.

16. Pourhoseingholi E, Pourhoseingholi MA, Comparison of Arch Index of flat foot and healthy foot in pre-school children. Thrita. 2013; 2(3): 15-8.

17. Aboitiz-Rivera CM. Conceptos actuales acerca del pie plano en los niños. Rev Mex Pediatr. 1999; 66(6): 257-9.

18. González AA, Rollán RA, Bonilla MC, Montes CA, Santamaría D, Obeso GM. Resultados del screening con podoscopio en 948 niños no seleccionados con especial referencia al pie cavo. An Esp Pediatr. 1996; 45: 579-82.

19. Espinoza-Navarro O, Olivares-Urquieta M, Palacios-Navarrete P, Robles-Flores N. Prevalencia de anomalías de pie en niños de enseñanza básica de entre 6 a 12 años, de colegios de la ciudad de Arica-Chile. Int J Morphol. 2013; 31(1): 162-8. 